\title{
Frequent Exertion and Frequent Standing at Work, by Industry and Occupation Group — United States, 2015
}

\author{
Taylor M. Shockey, $\mathrm{MPH}^{1}$; Sara E. Luckhaupt, $\mathrm{MD}^{1}$; Matthew R. Groenewold, PhD ${ }^{1}$; Ming-Lun Lu, PhD 2
}

Repeated exposure to occupational ergonomic hazards, such as frequent exertion (repetitive bending or twisting) and frequent standing, can lead to injuries, most commonly musculoskeletal disorders (1). Work-related musculoskeletal disorders have been estimated to cost the United States approximately $\$ 2.6$ billion in annual direct and indirect costs (2). A recent literature review provided evidence that prolonged standing at work also leads to adverse health outcomes, such as back pain, physical fatigue, and muscle pain (3). To determine which industry and occupation groups currently have the highest prevalence rates of frequent exertion at work and frequent standing at work, CDC analyzed data from the 2015 National Health Interview Survey (NHIS) Occupational Health Supplement (OHS) regarding currently employed adults in the United States. By industry, the highest prevalence of both frequent exertion and frequent standing at work was among those in the agriculture, forestry, fishing, and hunting industry group (70.9\%); by occupation, the highest prevalence was among those in the construction and extraction occupation group $(76.9 \%)$. Large differences among industry and occupation groups were found with regard to these ergonomic hazards, suggesting a need for targeted interventions designed to reduce workplace exposure.

NHIS is an annual, in-person, household interview survey of noninstitutionalized, U.S. civilian residents that has been continuously conducted since 1957 with the main purpose of monitoring the health of the U.S. population through assessment of a range of health topics and demographic characteristics.* The NHIS questionnaire contains a set of core questions with Household, Family, Sample Adult, and Sample Child components, which have remained relatively unchanged from 1997 through 2017. In addition, NHIS has sets of questions, known as Supplements, which vary each year depending on new public health data needs. In 2015, CDC's National Institute for

\footnotetext{
*https://www.cdc.gov/nchs/nhis/about_nhis.htm.
}

Occupational Safety and Health (NIOSH) sponsored an OHS to collect information on work-related health conditions as well as psychological and physical occupational exposures. The OHS questions were included in the Sample Adult questionnaire, which had a final, unconditional response rate of $55.2 \% .^{\dagger}$

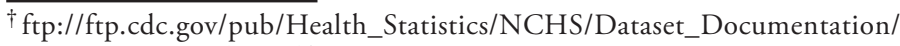
NHIS/2015/srvydesc.pdf.

\section{INSIDE}

7 Tobacco Product Use Among Military Veterans United States, 2010-2015

13 West Nile Virus and Other Nationally Notifiable Arboviral Diseases — United States, 2016

18 Antibiotics Dispensed to Privately Insured Pregnant Women with Urinary Tract Infections - United States, 2014

23 HIV Infection and HIV-Associated Behaviors Among Persons Who Inject Drugs - 20 Cities, United States, 2015

29 Status of HIV Epidemic Control Among Adolescent Girls and Young Women Aged 15-24 Years - Seven African Countries, 2015-2017

33 Recommendation of the Advisory Committee on Immunization Practices for Use of a Third Dose of Mumps Virus-Containing Vaccine in Persons at Increased Risk for Mumps During an Outbreak

39 Vital Signs: Trends and Disparities in Infant Safe Sleep Practices — United States, 2009-2015

47 Notices to Readers

49 QuickStats

Continuing Education examination available at https://www.cdc.gov/mmwr/cme/conted_info.html\#weekly.

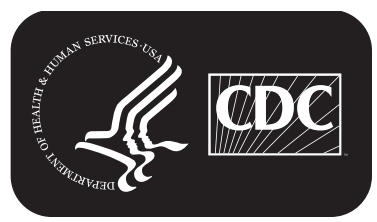

U.S. Department of Health and Human Services Centers for Disease Control and Prevention 
To determine industry and occupation, currently employed adult respondents were asked, in reference to the job they were working at during the week before the interview, "What kind of business or industry was this?" and "What kind of work were you doing?" Open-ended responses were recorded as text and subsequently coded by the U.S. Census Bureau into 4-digit codes derived from the 2012 North American Industrial Classification System (NAICS) industry groups and 2010 Standard Occupational Classification (SOC) occupation groups. To improve reliability of the statistical estimates, the detailed 4-digit industry and occupation groups were collapsed into 2-digit industry groups and occupation groups (based on the NAICS and SOC major groups ${ }^{\S}$ ). As part of the OHS, currently employed adults were asked two questions related to the ergonomics of their current job: "How often does your job involve repeated lifting, pushing, pulling, or bending?" and "How often does your job involve standing or walking around?" Responses to these questions were dichotomized into Often/ Always and Never/Seldom/Sometimes, to indicate frequent or infrequent exertion or standing, respectively. Responses to these two ergonomics questions were also used to create one dichotomous variable capturing respondents that reported both frequent exertion at work and frequent standing at work.

Among the 36,672 adult NHIS respondents, 19,456 were currently employed and considered for analyses. After

\footnotetext{
\$ https://www.census.gov/cgi-bin/sssd/naics/naicsrch?chart=2012 and https:// www.bls.gov/soc/major_groups.htm.
}

excluding 1,615 respondents who worked $<20$ hours per week, 187 respondents who did not provide adequate information on their hours worked in the previous week, and 190 respondents in military-specific occupations, the final analytic sample included 17,464 respondents $(89.8 \%$ of the currently employed adult respondents). Sample adults who worked more than 20 hours per week were more likely to be aged $<65$ years, men, and hold a college degree or higher; however, there was no difference in the distribution of frequent exertion and frequent standing by number of hours worked. Unadjusted prevalence of frequent exertion at work, frequent standing at work, and both frequent exertion and frequent standing at work were calculated by the 20 major industry groups and the 22 major occupation groups. The unadjusted prevalence estimates were obtained using statistical software. All analyses were weighted, and standard errors were adjusted to account for the survey design.

Overall, $39.5 \%$ of currently employed adults who work at least 20 hours per week reported both frequent exertion and frequent standing at work (Table 1). The prevalences of frequent exertion at work or frequent standing at work, or both frequent exertion at work and frequent standing at work were highest among men, persons aged 18-29 years, Hispanics, and adults with less than a high school diploma (Table 1).

Among the 20 major industry groups, the groups with the highest prevalence of both frequent exertion and frequent standing at work were agriculture, forestry, fishing, and hunting (70.9\%); construction (67.2\%); and accommodation and food

The MMWR series of publications is published by the Center for Surveillance, Epidemiology, and Laboratory Services, Centers for Disease Control and Prevention (CDC), U.S. Department of Health and Human Services, Atlanta, GA 30329-4027.

Suggested citation: [Author names; first three, then et al., if more than six.] [Report title]. MMWR Morb Mortal Wkly Rep 2018;67:[inclusive page numbers].

\section{Centers for Disease Control and Prevention Brenda Fitzgerald, MD, Director \\ Leslie Dauphin, PhD, Acting Associate Director for Science \\ Joanne Cono, MD, ScM, Director, Office of Science Quality \\ Chesley L. Richards, MD, MPH, Deputy Director for Public Health Scientific Services \\ Michael F. Iademarco, MD, MPH, Director, Center for Surveillance, Epidemiology, and Laboratory Services}

MMWR Editorial and Production Staff (Weekly)

Sonja A. Rasmussen, MD, MS, Editor-in-Chief

Charlotte K. Kent, PhD, MPH, Executive Editor Jacqueline Gindler, MD, Editor

Teresa F. Rutledge, Managing Editor

Douglas W. Weatherwax, Lead Technical Writer-Editor

Soumya Dunworth, PhD, Kristy Gerdes, MPH, Teresa M. Hood, MS, Technical Writer-Editors
Martha F. Boyd, Lead Visual Information Specialist

Maureen A. Leahy, Julia C. Martinroe, Stephen R. Spriggs, Tong Yang, Visual Information Specialists

Quang M. Doan, MBA, Phyllis H. King,

Paul D. Maitland, Terraye M. Starr, Moua Yang, Information Technology Specialists
MMWR Editorial Board

Timothy F. Jones, MD, Chairman

Matthew L. Boulton, MD, MPH Virginia A. Caine, MD

Katherine Lyon Daniel, PhD

Jonathan E. Fielding, MD, MPH, MBA

David W. Fleming, MD
William E. Halperin, MD, DrPH, MPH

King K. Holmes, MD, PhD

Robin Ikeda, MD, MPH

Rima F. Khabbaz, MD

Phyllis Meadows, PhD, MSN, RN

Jewel Mullen, MD, MPH, MPA
Jeff Niederdeppe, $\mathrm{PhD}$

Patricia Quinlisk, MD, MPH

Patrick L. Remington, MD, MPH

Carlos Roig, MS, MA

William L. Roper, MD, MPH

William Schaffner, MD 
TABLE 1. Weighted prevalence of frequent exertion at work, frequent standing at work, and both frequent exertion and frequent standing at work among adult U.S. workers, ${ }^{*}$ by demographic characteristics - National Health Interview Survey, 2015

\begin{tabular}{|c|c|c|c|c|c|c|c|c|c|}
\hline \multirow[b]{2}{*}{ Characteristic } & \multicolumn{3}{|c|}{$\begin{array}{l}\text { Both frequent exertion and frequent } \\
\text { standing at work }\end{array}$} & \multicolumn{3}{|c|}{ Frequent exertion at work } & \multicolumn{3}{|c|}{ Frequent standing at work } \\
\hline & $\begin{array}{c}\text { No. in } \\
\text { sample } \\
\text { exposed }\end{array}$ & $\begin{array}{l}\text { Weighted no. } \\
\text { in population }\end{array}$ & $\%(95 \% \mathrm{Cl})$ & $\begin{array}{c}\text { No. in } \\
\text { sample } \\
\text { exposed }\end{array}$ & $\begin{array}{l}\text { Weighted no. } \\
\text { in population }\end{array}$ & $\%(95 \% \mathrm{Cl})$ & $\begin{array}{c}\text { No. in } \\
\text { sample } \\
\text { exposed }\end{array}$ & $\begin{array}{l}\text { Weighted no. } \\
\text { in population }\end{array}$ & $\%(95 \% \mathrm{Cl})$ \\
\hline \multicolumn{10}{|l|}{ Sex } \\
\hline Men & 3,985 & $31,887,307$ & $44.1(42.7-45.5)$ & 4,235 & $33,946,823$ & $47.0(45.6-48.3)$ & 6,149 & $49,783,090$ & $68.8(67.5-70.2)$ \\
\hline Women & 2,997 & $20,897,950$ & $34.0(32.7-35.4)$ & 3,124 & $21,864,499$ & $35.6(34.2-37.0)$ & 5,520 & $39,328,061$ & $64.0(62.6-65.4)$ \\
\hline \multicolumn{10}{|l|}{ Age group (yrs) } \\
\hline $18-29$ & 1,682 & $14,707,666$ & $49.2(46.7-51.6)$ & 1,738 & $15,307,794$ & $51.2(48.7-53.7)$ & 2,593 & $22,663,742$ & $75.8(73.7-77.8)$ \\
\hline $30-44$ & 2,414 & $17,548,635$ & $39.0(37.4-40.7)$ & 2,538 & $18,448,479$ & $41.0(39.4-42.7)$ & 3,964 & $29,256,582$ & $65.1(63.4-66.7)$ \\
\hline $45-64$ & 2,603 & $19,130,997$ & $35.7(34.1-37.4)$ & 2,769 & $20,480,104$ & $38.3(36.6-39.9)$ & 4,517 & $34,073,378$ & $63.7(62.1-65.2)$ \\
\hline$\geq 65$ & 283 & $1,397,959$ & $26.3(22.5-30.0)$ & 314 & $1,574,945$ & $29.6(25.8-33.4)$ & 595 & $3,117,450$ & $58.6(54.4-62.8)$ \\
\hline \multicolumn{10}{|l|}{ Race/Ethnicity } \\
\hline White, non-Hispanic & 4,256 & $33,402,064$ & $38.4(37.2-39.7)$ & 4,466 & $35,094,556$ & $40.4(39.1-41.7)$ & 7,078 & $56,412,145$ & $64.9(63.6-66.1)$ \\
\hline Black, non-Hispanic & 919 & $6,736,754$ & $43.4(40.6-46.2)$ & 971 & $7,199,190$ & $46.4(43.6-49.2)$ & 1,555 & $11,046,554$ & $71.1(68.6-73.6)$ \\
\hline Other race, non-Hispanic & 363 & $2,340,168$ & $25.4(21.8-29.0)$ & 387 & $2,562,924$ & $27.8(24.1-31.4)$ & 691 & $4,767,859$ & $51.7(47.7-55.7)$ \\
\hline Hispanic & 1,444 & $10,306,271$ & $46.8(44.4-49.2)$ & 1,535 & $10,954,652$ & $49.8(47.4-52.2)$ & 2,345 & $16,884,593$ & 76.7 (74.7-78.7) \\
\hline \multicolumn{10}{|l|}{ Education level $^{\dagger}$} \\
\hline $\begin{array}{l}\text { Less than high school } \\
\text { diploma }\end{array}$ & 910 & $6,425,171$ & $59.5(56.0-63.0)$ & 967 & $6,840,231$ & $63.4(60.1-66.6)$ & 1,269 & $9,036,138$ & $83.7(81.4-86.0)$ \\
\hline High school diploma/GED & 2,138 & $16,679,503$ & $56.6(54.5-58.7)$ & 2,249 & $17,558,718$ & $59.6(57.4-61.7)$ & 3,000 & $23,231,939$ & $78.8(77.1-80.5)$ \\
\hline Some college & 2,612 & $19,898,969$ & $47.3(45.4-49.2)$ & 2,744 & $21,027,565$ & $50.0(48.1-51.9)$ & 3,986 & $30,423,401$ & $72.3(70.7-73.8)$ \\
\hline $\begin{array}{l}\text { Bachelor's degree or } \\
\text { higher }\end{array}$ & 1,303 & $9,513,585$ & $18.7(17.4-19.9)$ & 1,379 & $10,116,181$ & $19.8(18.6-21.1)$ & 3,384 & $26,079,808$ & $51.2(49.4-52.9)$ \\
\hline $\begin{array}{l}\text { All currently employed } \\
\text { adults }\end{array}$ & 6,982 & $52,785,257$ & $39.5(38.5-40.5)$ & 7,359 & $55,811,322$ & $41.7(40.7-42.7)$ & 11,669 & $89,111,151$ & $66.6(65.6-67.6)$ \\
\hline
\end{tabular}

Abbreviations: $\mathrm{Cl}$ = confidence interval; GED = General Educational Development.

* The survey sample consisted of 17,464 U.S. workers aged $\geq 18$ years who worked at least 20 hours per week.

† Education level only shown for persons aged $\geq 25$ years.

services $(57.7 \%)$ (Table 2$)$. These same three industry groups also had the highest prevalence rates of frequent exertion at work and frequent standing at work considered separately. The finance and insurance industry group had the lowest prevalence rates of all three exposures (Table 2). Among the 22 major occupation groups, the groups with the highest prevalence of both frequent exertion and frequent standing at work were construction and extraction $(76.9 \%)$; farming, fishing, and forestry (75.5\%); and building and grounds cleaning and maintenance $(74.0 \%)$ (Table 3$)$. These same three occupation groups also had the highest prevalence rates for frequent exertion at work. The food preparation and serving related occupation group (97.2\%) had the highest prevalence of frequent standing at work. The computer and mathematical occupation group had the lowest prevalence rate of the combined exposures of frequent exertion and frequent standing at work $(4.6 \%)$ (Table 3$)$.

\section{Discussion}

This is the first CDC report to evaluate exposure to frequent exertion and frequent standing at work among U.S. employed adults in all industries and occupations. The prevalence of exposure to both of these ergonomic hazards was higher among agricultural and construction workers than among workers in all other industries. A previous study using the U.S. Department of Labor's Occupational Information Network database found that of 10 detailed occupation categories evaluated with regard to self-reported bending or twisting at work, half were construction-related, which is consistent with the findings from this study (4). In addition, previous research using NHIS data that evaluated musculoskeletal disorders among agricultural workers found that low back pain was the most prevalent musculoskeletal disorder. That study also found that agricultural workers had a significantly higher prevalence of upper extremity pain compared with all other industries (5). Research has shown that agricultural and construction work are physically demanding, as these industries often require manual material handling, repetitive exertions, awkward body postures, and use of machinery that causes whole body vibration (4-7).

Approximately two thirds of all workers reported frequent standing at work. The industry and occupation groups that reported high prevalence rates of frequent exertion (e.g., farming, construction, and food services) also tended to report high prevalence rates of frequent standing, possibly because bending, pushing, pulling, and lifting commonly co-occur with standing. Several industry and occupation groups, such as education 
TABLE 2. Weighted prevalence of frequent exertion at work, frequent standing at work, and both frequent exertion and frequent standing at work among adult U.S. workers, ${ }^{*}$ by industry group — National Health Interview Survey, 2015

\begin{tabular}{|c|c|c|c|c|c|c|c|c|c|}
\hline \multirow[b]{2}{*}{ Industry group ${ }^{\dagger}$} & \multicolumn{3}{|c|}{$\begin{array}{l}\text { Both frequent exertion and frequent } \\
\text { standing at work }\end{array}$} & \multicolumn{3}{|c|}{ Frequent exertion at work } & \multicolumn{3}{|c|}{ Frequent standing at work } \\
\hline & $\begin{array}{l}\text { No. in } \\
\text { sample } \\
\text { exposed }\end{array}$ & $\begin{array}{l}\text { Weighted no. } \\
\text { in population }\end{array}$ & $\%(95 \% \mathrm{Cl})$ & $\begin{array}{l}\text { No. in } \\
\text { sample } \\
\text { exposed }\end{array}$ & $\begin{array}{l}\text { Weighted no. } \\
\text { in population }\end{array}$ & $\%(95 \% \mathrm{Cl})$ & $\begin{array}{l}\text { No. in } \\
\text { sample } \\
\text { exposed }\end{array}$ & $\begin{array}{l}\text { Weighted no. } \\
\text { in population }\end{array}$ & $\%(95 \% \mathrm{Cl})$ \\
\hline $\begin{array}{l}\text { Agriculture, Forestry, } \\
\text { Fishing, and Hunting }\end{array}$ & 199 & $1,168,731$ & $70.9(63.2-78.5)$ & 213 & $1,241,068$ & $75.2(68.0-82.5)$ & 238 & $1,428,182$ & $86.6(81.2-92.0)$ \\
\hline Construction & 741 & $5,673,721$ & $67.2(63.5-70.8)$ & 782 & $5,959,974$ & $70.6(67.0-74.2)$ & 900 & $7,041,656$ & $83.4(80.4-86.3)$ \\
\hline $\begin{array}{l}\text { Accommodation and } \\
\text { Food Services }\end{array}$ & 703 & $5,272,820$ & $57.7(53.6-61.7)$ & 712 & $5,317,174$ & $58.2(54.1-62.2)$ & 1,093 & $8,459,753$ & $92.5(90.6-94.4)$ \\
\hline Retail Trade & 955 & $7,504,966$ & $54.6(51.1-58.2)$ & 977 & $7,682,555$ & $55.9(52.4-59.4)$ & 1,403 & $11,235,663$ & $81.7(79.3-84.1)$ \\
\hline $\begin{array}{l}\text { Arts, Entertainment, } \\
\text { and Recreation }\end{array}$ & 138 & $1,165,969$ & $50.1(41.3-59.0)$ & 143 & $1,214,309$ & $52.2(43.8-60.6)$ & 244 & $1,869,437$ & $80.4(74.7-86.0)$ \\
\hline $\begin{array}{l}\text { Health Care and Social } \\
\text { Assistance }\end{array}$ & 1,128 & $8,186,368$ & $45.9(43.2-48.6)$ & 1,171 & $8,486,195$ & $47.6(44.9-50.3)$ & 1,858 & $13,360,776$ & $74.9(72.7-77.1)$ \\
\hline $\begin{array}{l}\text { Administrative and } \\
\text { support and Waste } \\
\text { management and } \\
\text { remediation services }\end{array}$ & 350 & $2,780,964$ & $45.7(41.2-50.1)$ & 374 & $2,955,167$ & $48.5(43.9-53.1)$ & 539 & $4,230,331$ & $69.4(65.6-73.3)$ \\
\hline Manufacturing & 818 & $6,742,939$ & $44.7(41.8-47.7)$ & 872 & $7,299,479$ & $48.4(45.4-51.4)$ & 1,198 & $10,054,756$ & $66.7(63.7-69.6)$ \\
\hline $\begin{array}{l}\text { Other service (except } \\
\text { Public Administration) }\end{array}$ & 372 & $2,875,412$ & $44.1(39.5-48.7)$ & 389 & $3,003,845$ & $46.0(41.6-50.5)$ & 624 & $4,830,228$ & $74.0(69.8-78.3)$ \\
\hline $\begin{array}{l}\text { Transportation and } \\
\text { warehousing }\end{array}$ & 294 & $2,238,125$ & $43.7(38.5-49.0)$ & 352 & $2,781,765$ & $54.4(49.3-59.5)$ & 383 & $2,882,926$ & $56.4(51.2-61.5)$ \\
\hline Wholesale trade & 181 & $1,563,819$ & $40.2(33.3-47.2)$ & 195 & $1,683,560$ & $43.3(36.4-50.2)$ & 274 & $2,349,503$ & $60.4(54.3-66.6)$ \\
\hline Utilities & 54 & 283,706 & $27.7(19.3-36.1)$ & 56 & 288,971 & $28.2(19.8-36.6)$ & 96 & 593,375 & $57.9(47.4-68.5)$ \\
\hline Mining & 60 & 217,846 & $27.0(19.3-34.6)$ & 63 & 226,742 & $28.1(20.2-36.0)$ & 88 & 470,569 & $58.3(46.0-70.5)$ \\
\hline $\begin{array}{l}\text { Real Estate and Rental } \\
\text { and Leasing }\end{array}$ & 107 & 745,525 & $26.2(20.2-32.2)$ & 110 & 794,548 & $27.9(21.7-34.2)$ & 242 & $1,773,803$ & $62.4(55.2-69.6)$ \\
\hline Information & 84 & 701,050 & $23.7(17.7-29.6)$ & 96 & 816,293 & $27.5(21.2-33.9)$ & 157 & $1,305,710$ & $44.0(37.1-51.0)$ \\
\hline Public administration & 218 & $1,594,215$ & $23.0(19.6-26.4)$ & 234 & $1,706,227$ & $24.6(21.1-28.1)$ & 525 & $3,794,816$ & $54.8(50.4-59.2)$ \\
\hline Education services & 390 & $2,698,347$ & $22.7(19.9-25.5)$ & 402 & $2,778,152$ & $23.4(20.5-26.2)$ & 1,187 & $8,599,529$ & $72.3(69.4-75.2)$ \\
\hline $\begin{array}{l}\text { Professional, scientific, } \\
\text { and technical } \\
\text { services }\end{array}$ & 134 & $1,026,452$ & $9.8(7.6-12.0)$ & 153 & $1,159,929$ & $11.1(8.8-13.3)$ & 404 & $3,149,955$ & $30.1(26.5-33.6)$ \\
\hline Finance and Insurance & 55 & 342,473 & $5.0(3.2-6.8)$ & 64 & 413,560 & $6.0(3.9-8.2)$ & 209 & $1,651,592$ & $24.1(20.3-27.9)$ \\
\hline $\begin{array}{l}\text { All currently } \\
\text { employed adults }\end{array}$ & ,982 & $2,785,257$ & $39.5(38.5-40.5)$ & 359 & $55,811,322$ & $41.7(40.7-42.7)$ & 11,669 & $89,111,151$ & $66.6(65.6-67.6)$ \\
\hline
\end{tabular}

Abbreviation: $\mathrm{Cl}=$ confidence interval.

* The survey sample consisted of 17,464 U.S. workers aged $\geq 18$ years who worked at least 20 hours per week.

$\dagger$ The Management of Companies and Enterprises industry group was removed from the results because the cell size was $<10$ and did not meet the National Center for Health Statistics' standards of reliability.

and protective services, reported a high prevalence of frequent standing at work with a low prevalence of frequent exertion at work compared with other industry and occupation groups.

Recent studies have emphasized health risks associated with excessive sitting during the workday (8); however, excessive standing on the job also has been linked to adverse health outcomes (9). A systematic review of peer-reviewed articles on musculoskeletal symptoms and occupational standing as the main exposure variable found that occupational standing is associated with low back pain; however, associations with lower and upper extremity symptoms were inconclusive (9). More research is needed to understand how to balance time spent sitting and standing while at work.

The findings in this report are subject to at least four limitations. First, because NHIS data are cross-sectional, it is not possible to make causal inferences. Second, because NHIS data are self-reported, they are subject to recall or social desirability bias. Third, the intermediate exposure categories (Often, Sometimes, and Seldom) rely on subjective assessment of frequency. Finally, collapsing the detailed industry and occupation groups into the major industry and occupation groups might have aggregated employees with different working conditions.

Healthy People 2020 has an objective to "reduce rate of injury and illness cases involving days away from work due to overexertion and repetitive motion," by at least $10 \% .9$ NIOSH has developed educational resources on a variety of ergonomic issues. ${ }^{* *}$ For example, NIOSH provides a

\footnotetext{
https://www.healthypeople.gov/2020/topics-objectives/topic/occupationalsafety-and-health/objectives.

** https://www.cdc.gov/niosh/topics/ergonomics/default.html.
} 
TABLE 3. Weighted prevalence of frequent exertion at work, frequent standing at work, and both frequent exertion and frequent standing at work among adult U.S. workers, ${ }^{*}$ by occupation group - National Health Interview Survey, 2015

\begin{tabular}{|c|c|c|c|c|c|c|c|c|c|}
\hline \multirow[b]{2}{*}{ Occupation group } & \multicolumn{3}{|c|}{$\begin{array}{l}\text { Both frequent exertion and } \\
\text { frequent standing at work }\end{array}$} & \multicolumn{3}{|c|}{ Frequent exertion at work } & \multicolumn{3}{|c|}{ Frequent standing at work } \\
\hline & $\begin{array}{l}\text { No. in } \\
\text { sample } \\
\text { exposed }\end{array}$ & $\begin{array}{l}\text { Weighted no. } \\
\text { in population }\end{array}$ & $\%(95 \% \mathrm{Cl})$ & $\begin{array}{c}\text { No. in } \\
\text { sample } \\
\text { exposed }\end{array}$ & $\begin{array}{l}\text { Weighted no. } \\
\text { in population }\end{array}$ & $\%(95 \% \mathrm{Cl})$ & $\begin{array}{l}\text { No. in } \\
\text { sample } \\
\text { exposed }\end{array}$ & $\begin{array}{l}\text { Weighted no. } \\
\text { in population }\end{array}$ & $\%(95 \% \mathrm{Cl})$ \\
\hline $\begin{array}{l}\text { Construction and } \\
\text { Extraction }\end{array}$ & 685 & $4,856,232$ & $76.9(73.2-80.6)$ & 718 & $5,077,403$ & $80.4(76.8-84.1)$ & 793 & $5,739,639$ & $90.9(88.7-93.2)$ \\
\hline $\begin{array}{l}\text { Farming, Fishing, and } \\
\text { Forestry }\end{array}$ & 129 & 731,178 & $75.5(65.3-85.8)$ & 133 & 749,387 & $77.4(67.3-87.4)$ & 147 & 888,366 & 91.7 (86.9-96.6) \\
\hline $\begin{array}{l}\text { Building and Grounds } \\
\text { Cleaning and } \\
\text { Maintenance }\end{array}$ & 518 & $3,495,764$ & $74.0(69.9-78.1)$ & 532 & $3,605,331$ & $76.3(72.3-80.3)$ & 658 & $4,332,921$ & $91.7(88.7-94.7)$ \\
\hline $\begin{array}{l}\text { Installation, } \\
\text { Maintenance, and } \\
\text { Repair }\end{array}$ & 436 & $1,290,688$ & $73.0(68.4-77.6)$ & 451 & $3,611,498$ & 75.5 (71.0-79.9) & 516 & $4,238,754$ & $88.6(85.1-92.1)$ \\
\hline $\begin{array}{l}\text { Food Preparation and } \\
\text { Serving Related }\end{array}$ & 587 & $4,277,608$ & $65.7(61.1-70.2)$ & 591 & $4,304,915$ & $66.1(61.5-70.7)$ & 853 & $6,336,456$ & $97.2(96.1-98.4)$ \\
\hline Production & 704 & $5,615,533$ & $65.2(61.5-69.0)$ & 738 & $5,884,574$ & $68.3(64.7-72.0)$ & 940 & $7,310,817$ & $84.9(82.2-87.6)$ \\
\hline Healthcare Support & 285 & $1,965,904$ & $62.2(56.2-68.1)$ & 291 & $2,010,000$ & $63.6(57.6-69.5)$ & 395 & $2,769,227$ & $87.6(83.5-91.7)$ \\
\hline $\begin{array}{l}\text { Transportation and } \\
\text { Material Moving }\end{array}$ & 540 & $4,244,701$ & $55.2(51.0-59.5)$ & 642 & $5,187,351$ & 67.5 (63.4-71.6) & 648 & $5,182,206$ & $67.4(63.4-71.4)$ \\
\hline $\begin{array}{l}\text { Healthcare } \\
\text { Practitioners and } \\
\text { Technical }\end{array}$ & 527 & $4,272,338$ & $53.1(49.2-57.1)$ & 543 & $4,375,067$ & $54.4(50.3-58.5)$ & 874 & $6,955,460$ & $86.5(84.1-88.9)$ \\
\hline $\begin{array}{l}\text { Personal Care and } \\
\text { Service }\end{array}$ & 278 & $2,072,693$ & $52.2(46.1-58.3)$ & 290 & $2,135,012$ & $53.8(47.7-59.8)$ & 467 & $3,448,249$ & $86.8(83.3-90.4)$ \\
\hline Sales and Related & 657 & $5,313,669$ & $39.3(35.7-42.9)$ & 675 & $5,484,798$ & $40.6(37.0-44.2)$ & 1,194 & $10,006,503$ & 73.9 (70.9-76.8) \\
\hline Protective Service & 119 & 907,995 & $35.0(28.2-41.8)$ & 123 & 925,096 & $35.6(28.7-42.6)$ & 280 & $2,198,270$ & $84.7(79.5-89.8)$ \\
\hline $\begin{array}{l}\text { Education, Training, } \\
\text { and Library }\end{array}$ & 267 & $2,057,303$ & $25.2(21.5-28.9)$ & 274 & $2,104,193$ & $25.8(22.1-29.5)$ & 891 & $6,717,390$ & $82.2(79.2-85.3)$ \\
\hline $\begin{array}{l}\text { Office and } \\
\text { Administrative } \\
\text { Support }\end{array}$ & 528 & $3,847,255$ & $24.3(21.8-26.8)$ & 567 & $4,166,405$ & $26.3(23.7-28.9)$ & 996 & $7,528,768$ & $47.5(44.8-50.2)$ \\
\hline $\begin{array}{l}\text { Arts, Design, } \\
\text { Entertainment, } \\
\text { Sports and Media }\end{array}$ & 74 & 579,465 & $22.8(16.9-28.7)$ & 80 & 631,237 & $24.8(18.9-30.8)$ & 171 & $1,145,550$ & $45.1(38.3-51.8)$ \\
\hline Management & 418 & $3,258,402$ & $22.5(19.9-25.1)$ & 444 & $3,441,037$ & $23.7(21.1-26.4)$ & 965 & $7,543,484$ & $52.0(49.0-55.0)$ \\
\hline $\begin{array}{l}\text { Community and } \\
\text { Social Services }\end{array}$ & 52 & 423,039 & $15.7(10.0-21.5)$ & 53 & 444,643 & 16.5 (10.7-22.4) & 217 & $1,448,680$ & $53.9(47.1-60.7)$ \\
\hline $\begin{array}{l}\text { Life, Physical, and } \\
\text { Social Science }\end{array}$ & 31 & 176,272 & $12.1(6.1-18.0)$ & 34 & 220,958 & $15.1(8.3-21.9)$ & 107 & 734,098 & $50.2(40.9-59.5)$ \\
\hline $\begin{array}{l}\text { Architecture and } \\
\text { Engineering }\end{array}$ & 35 & 337,561 & $10.2(6.1-14.3)$ & 39 & 366,910 & $11.1(6.9-15.3)$ & 144 & $1,318,074$ & $39.8(33.4-46.3)$ \\
\hline $\begin{array}{l}\text { Business and Financial } \\
\text { Operations }\end{array}$ & 76 & 594,080 & $7.9(5.7-10.1)$ & 89 & 696,504 & $9.2(6.9-11.6)$ & 242 & $1,967,945$ & $26.1(22.5-29.7)$ \\
\hline $\begin{array}{l}\text { Computer and } \\
\text { Mathematical }\end{array}$ & 29 & 232,386 & $4.6(2.6-6.6)$ & 41 & 336,596 & $6.7(4.1-9.2)$ & 116 & 949,751 & $18.8(14.8-22.8)$ \\
\hline Legal & 7 & 32,566 & — & 11 & 52,407 & $3.1(0.8-5.4)^{\dagger}$ & 55 & 350,543 & $20.6(13.5-27.6)$ \\
\hline $\begin{array}{l}\text { All currently } \\
\text { employed adults }\end{array}$ & 6,982 & $52,785,257$ & $39.5(38.5-40.5)$ & 7,359 & $55,811,322$ & $41.7(40.7-42.7)$ & 11,669 & $89,111,151$ & $66.6(65.6-67.6)$ \\
\hline
\end{tabular}

Abbreviation: $\mathrm{Cl}=$ confidence interval.

* The survey sample consisted of 17,464 U.S. workers aged $\geq 18$ years who worked at least 20 hours per week.

† Estimate has a relative standard error $>30 \%$ and $<50 \%$ and should be used with caution because it does not meet the National Center for Health Statistics' standards of reliability.

$\S$ Estimate had a cell size $<10$ and was removed from the results because it did not meet the National Center for Health Statistics' standards of reliability.

demonstration guide on ergonomic principles including how to maintain neutral postures when working, how to select the appropriate hand tools, and how to prevent fatigue failure of the vertebrae. In addition, NIOSH offers ergonomic guidelines for manual material handling, a primer for creating a workplace ergonomic programs, and ergonomic interventions by specific industry, including agriculture and construction. ${ }^{\dagger \dagger}$ Because ergonomic hazards are risk factors for work-related musculoskeletal disorders, continued research is necessary to develop a better understanding of these hazards and to create interventions aimed at reducing them $(2,8-10)$.

\footnotetext{
$\dagger \dagger$ https://www.cdc.gov/niosh/docs/2001-111/ and https://www.cdc.gov/niosh/ docs/2007-122/.
} 


\section{Conflict of Interest}

No conflicts of interest were reported.

\begin{abstract}
${ }^{1}$ Division of Surveillance, Hazard Evaluations, and Field Studies, National Institute for Occupational Safety and Health, CDC; ${ }^{2}$ Division of Applied Research and Technology, National Institute for Occupational Safety and Health, CDC.
\end{abstract}

Corresponding author: Taylor M. Shockey, tshockey@cdc.gov, 513-841-4239.

\section{References}

1. Bernard BP. Musculoskeletal disorders and workplace factors: a critical review of epidemiologic evidence for work-related musculoskeletal disorders of the neck, upper extremity, and low back. DHHS (NIOSH) publication no. 97-141;1997.

2. Bhattacharya A. Costs of occupational musculoskeletal disorders (MSDs) in the United States. Int J Ind Ergon 2014;44:448-54. https://doi. org/10.1016/j.ergon.2014.01.008

3. Waters TR, Dick RB. Evidence of health risks associated with prolonged standing at work and intervention effectiveness. Rehabil Nurs 2015;40:148-65. https://doi.org/10.1002/rnj.166

4. Tak S, Calvert GM. The estimated national burden of physical ergonomic hazards among US workers. Am J Ind Med 2011;54:395-404. https:// doi.org/10.1002/ajim.20883

5. Lee SJ, Tak S, Alterman T, Calvert GM. Prevalence of musculoskeletal symptoms among agricultural workers in the United States: an analysis of the National Health Interview Survey, 2004-2008. J Agromed 2014;19:268-80. https://doi.org/10.1080/1059924X.2014.916642

6. Boschman JS, van der Molen HF, Sluiter JK, Frings-Dresen MH. Occupational demands and health effects for bricklayers and construction supervisors: a systematic review. Am J Ind Med 2011;54:55-77. https:// doi.org/10.1002/ajim.20899

7. Choi SD, Hudson L, Kangas P, Jungen B, Maple J, Bowen C. Occupational ergonomic issues in highway construction surveyed in Wisconsin, United States. Ind Health 2007;45:487-93. https://doi. org/10.2486/indhealth.45.487

8. van Uffelen JGZ, Wong J, Chau JY, et al. Occupational sitting and health risks: a systematic review. Am J Prev Med 2010;39:379-88. https://doi.org/10.1016/j.amepre.2010.05.024

9. Coenen P, Willenberg L, Parry S, et al. Associations of occupational standing with musculoskeletal symptoms: a systematic review with meta-analysis. Br J Sports Med 2016;0:1-9.

10. da Costa BR, Vieira ER. Risk factors for work-related musculoskeletal disorders: a systematic review of recent longitudinal studies. Am J Ind Med 2010;53:285-323.

\section{Summary}

What is already known about this topic?

Occupational ergonomic hazards are risk factors for negative health outcomes such as musculoskeletal disorders. Previous research has found that employees in the agricultural and construction sectors experience high rates of musculoskeletal disorders and other injuries because of the physical nature of the work and has also found that workers in the construction and agricultural sectors have high prevalence rates of exertion including bending, lifting, pushing, and pulling.

What is added by this report?

Analysis of data from the National Health Interview Survey to examine two ergonomic hazards among currently employed adults who work at least 20 hours per week in 20 major industry groups and 22 major occupation groups found a $41.7 \%$ prevalence of frequent exertion (repeated lifting, pushing, pulling, or bending) at work and a $66.6 \%$ prevalence of frequent standing at work. A wide range in prevalence for these ergonomic hazards was observed among the industry and occupation groups.

What are the implications for public health practice?

Large differences in prevalence of frequent exertion at work and frequent standing at work exist among the major industry and occupation groups. Identification of workers with the highest prevalences of exposure to these two ergonomic hazards can inform the targeting of interventions. 